\title{
Aportes de Paulo Freire a la Investigación y a la Lectura Crítica
}

\author{
Paulo Freire's Contributions to the Research and Critical \\ Reading
}

\author{
Jesús Morales * \\ Universidad de Los Andes, Colombia
}

\begin{abstract}
Los aportes de Paulo Freire a la educación del siglo XXI han conseguido significativa aceptación por su invaluable vigencia. Sus implicaciones en la enseñanza de la investigación y la lectura crítica han sido reconsideradas por la evidente promoción de habilidades cognitivas desde las cuales posibilitarle al sujeto romper con los esquemas impositivos de una educación reproductora. Atendiendo a la relevancia de la obra de Freire, se realiza una revisión de los aspectos significativos en los que dejan entrever a la lectura crítica y a la investigación como herramientas que persiguen la transformación y el desarrollo de habilidades sociales y competencias educativas como herramientas fundamentales el ejercicio de valores democráticos, cuya contribución implícita no es otra que la educación ciudadana necesaria para la práctica de valores tales como: la autonomía, el respeto la tolerancia, la responsabilidad y el reconocimiento del otro como co-constructor y parte integral de su experiencia transformadora. Se exponen los esquemas de investigación y de lectura crítica propuestos por Freire, en un intento por destacar aspectos elementales para la formación académica del presente siglo, como lo son: el desarrollo del pensamiento crítico, la promoción de la indagación como habilidad necesaria para problematizar el mundo y el uso de la criticidad como herramienta al servicio de una recurrente objeción y reflexión sobre la realidad teniendo como elementos determinantes la autonomía y la libertad, como medios necesarios para accionar de manera trascendental y efectiva en su propio contexto.
\end{abstract}

Descritores: Investigación; Lectura crítica; Formación; Educación; Justicia social.

Paulo Freire's contributions to the education of XXI century have attained significant acceptance owing to its invaluable validity. Its implications in the teaching of researching and critical reading have been taking into account because of its promotion of cognitive abilities which allow the individual to achieve break the imposing schemes of a reproductive education. Attending to the relevance of Freire's work, a revision of the significant aspects in those that hint at critical reading and research as tools that pursue the transformation and improvement of social abilities and educational competences as fundamental tools for exercising democratic values which implicit contribution is the civic education necessary in order to practice values such as: autonomy, respect, tolerance, responsibility and to recognition of the other as a constructor and integral part of her or his transformation experience is done. Freire's proposal of research schemes and critical reading are exposed in an intent to highlight elemental aspects of academic formation in this present century, such as: the development of critical thinking, the promotion of inquiry as a necessary ability in order to question the world and the use of the critique as a tool to the service of a recurrent objection and reflection about reality keeping as determining elements the autonomy and the freedom as essential means to operate in transcendental and effective ways their own context.

Keywords: Research; Reading; Training; Education; Social justice.

*Contacto: lectoescrituraula@gmail.com

ISSN: 2254-3139

www.rinace.net/riejs/

revistas.uam.es/riejs
Recibido: $\quad 6$ de septiembre 2018

$1^{\text {a }}$ Evaluación: 9 de octubre 2018

$2^{\text {a }}$ Evaluación: 18 de octubre 2018

Aceptado: 3 de noviembre 2018 


\section{Introducción}

La enseñanza de la lectura en el siglo XXI ha conseguido en Freire uno de los referentes más importantes, pues sus aportes se han centrado en determinar que el acto de acercarse al conocimiento no responde a un proceso automático, sin reflexión, en solitario ni ingenuo, sino colectivo en el que los más experimentados son capaces de interactuar con otros con el propósito de llevarlos a descubrir nuevas maneras de construcción el mundo y la realidad, a través de la promoción y uso del pensamiento en sus modos críticos, reflexivos y analíticos como instrumentos fundamentales para transformar de manera autónoma el saber, así como buscar razones y explicaciones sobre lo que acontece en su propio contex to (Freire, 1991).

A partir de esta premisa es posible afirmar que, en Freire la concepción de lectura y en especial de lectura crítica, se aprecia como una herramienta al servicio de la liberación del hombre, es decir, como un medio a partir del cual percibir intenciones de dominación como resultado de la manipulación histórica, sino además como un proceso cuya continuidad favorece el falsear el conocimiento tradicional como parte de su propia construcción responsable del mundo y de la cual va a depender la elaboración de reflexiones firmes y sólidas que le posibiliten para ejercer transformaciones pertinentes, efectivas y trascendentales en el contex to del que hace parte.

Por tal motivo leer como proceso social entraña el potencial para modificar las estructuras de dominación que históricamente han aquejado al hombre, posibilitándole el desarrollo de habilidades propias del pensamiento que le cooperen en la búsqueda y detección de contradicciones en el conocimiento acumulado (Zemelman, 2005) con el propósito de ir más allá en un proceso de profundización que lo prepare para luchar contra las fuerzas opresivas que intentan llevarlo a la repetición de modelos preestablecidos, que la mayoría de las veces sino en todas, responden a recetas carentes de relevancia, pertinencia y adecuación a las necesidades del sujeto y su realidad (Morales, 2017).

Lo expuesto implica entonces que, el leer viene a constituirse según Freire en un instrumento para el desarrollo de la sensibilidad individual cuyos cometidos intentan llevar al sujeto a la construcción conjunta de mejores formas de vida, en un operar activo fundado en el uso de la autonomía y de la libertad de pensamiento, como medios que le permitan problematizar el mundo y buscar recurrentemente de razones y explicaciones desde las que le sea posible según Freire (1991) el "abrir los ojos a la exploración de su propia realidad, de la que muchas veces solo tiene nociones o simplemente ignora como resultado de una cultura acrítica impuesta históricamente” (p. 15).

De esta manera, la obra de Freire ha sido asumida por los críticos de la educación impositiva y reproductora como una alternativa para la transformación integral del ser humano, que procura mejorar la condición humana al llevar al hombre a explorarse y, por ende, a conocerse de manera reflexiva asumiendo un rol protagónico que lo lleve a desarrollar modos de pensamiento y de acción cónsonos que procuren la transformación propia y de su realidad (Daros, 2009; Freire, 2002), como procesos permanentes, constantes y extensibles para la vida (Torres, 2006). Para ello parte de sus cometidos se han enfocado en el fortalecimiento de un pensamiento acucioso con tendencia hacia la criticidad y a la reflexión como habilidades a partir de las cuales problematizar su realidad en una búsqueda caracterizada por la elaboración de hipótesis e interrogantes, predicciones e inferencias como actividades necesarias para responder a las interrogantes 
que emergen de su relación con el mundo (Argudin, 1995; Freire, 2002; Goodman, 1996; Smith, 1983; Solé, 1997; Ulloa, s/f).

En atención a estos planteamientos, el presente ensayo tiene como propósito realizar una revisión de las apreciaciones y aportes desarrollados por Paulo Freire sobre la lectura crítica y la investigación como actividades indispensables en todo proceso educativo, entre cuyas funciones se encuentra la creación de condiciones que favorezcan la consolidación de importantes habilidades cognitivas y sociales, que por medio del acompañamiento como proceso propiamente educativo e indispensable que lleve al individuo a una liberación consciente y reflexiva como condición fundamental para desarrollar su potencial en sociedad (Daros, 2009; Freire, 1967).

\section{Disertación temática}

El aprendizaje como un proceso que prepara al ciudadano para la vida ha conseguido en la teoría crítica de la educación y en las concepciones recientes de la alfabetización académica (Freire, 2004; Torres, 2006) los fundamentos que defienden la idea de promover el desarrollo del pensamiento como el instrumento desde el cual operar sobre el mundo de manera autónoma y responsable. En atención a esta premisa, la lectura crítica se ha posicionado como una herramienta enfocada en promover el desarrollo de habilidades cognitivas que posicionan al hombre como sujeto histórico capaz de asumir postura desde la crítica y la reflexión de su entorno con el propósito de generar nuevas aportaciones que diversifiquen la comprensión del mundo.

En este sentido, los aportes de Paulo Freire vistos desde el enfoque pedagógico y social, se han configurado en una respuesta contestaría frente a las prácticas e imposiciones emanadas de los sistemas educativos tradicionales cuyo objetivo no ha sido otro que someter al sujeto a los efectos de las fuerzas históricas de dominación. Por ende, su pensamiento libertador y fecundo ha dinamizado el proceso educativo valiéndose de la inserción de actividades reflexivas, encaminadas hacia la trasformación de los actos de enseñar y aprender, al despertar en sus integrantes el sentido crítico necesario para generar cambios medulares en los procesos de comprensión del mundo y su carácter dinámico. Esta pretensión como se expone a lo largo de sus diversas obras, ha perseguido la construcción de condiciones para el desarrollo del pensamiento como medio para que el hombre se haga más humano (Daros, 2009; Delors, 1994; Freire, 2002).

En este sentido, el pensamiento pedagógico de Freire ha conseguido aceptación por el hecho de revolucionar el proceso educativo como escenario estratégico desde el cual promover el redescubrimiento del hombre y su conocimiento profundo como acciones de las que dependen rompimiento de las imposiciones y los efectos nocivos derivados de los diversos esquemas de dominación. Lo expuesto ha significado un giro en la concepción del educador quien se asume como actor elemental, sobre el cual recae el compromiso de guiar al ciudadano hacia nuevas formas de vida, construidas como resultado del operar crítico y reflexivo sobre su propia realidad.

En apoyo a esta posición Freire ha planteado que los cometidos de la educación deben enfocarse en llevar al hombre progresivamente a profundizar sobre sus orígenes, con la finalidad de que éste desarrolle su conciencia histórica desde la cual ser capaz de manejar objetiva y racionalmente su interacción con el mundo y combatir las manipulaciones sociales, políticas e ideológicas (Freire, 2002). Esta premisa desde la formación ciudadana 
implica el rompimiento de relaciones represivas y los efectos de la imposición histórica, que han conseguido en la educación el instrumento para cercenar la libertad, la autonomía y la responsabilidad como valores suficientes para preparar al ciudadano para la vida (Fons, 2006) la trasformación y el accionar social, así como la reformulación de ideas vistas hasta ahora de forma monádica y para la reconstrucción activa del mundo desde una actitud crítica y reflexiva que le permita según López (2013) el "enfrentar los retos de la vida y el ir más allá, hacia la búsqueda de alternativas verdaderas y reales que den cabida a la transformación" (p.47).

Estos planteamientos demuestran que la obra de Freire constituye una contrarespuesta a los modelos educativos tradicionalmente impositivos que, por su rigidez, han cerrado la posibilidad de desarrollar el pensamiento como instrumento para el ejercicio de la libertad (Valera y Escobar, s/f). De allí que sus apreciaciones educativas además de generar polémica dentro del campo de la pedagogía, también han representado una invitación a la formación de ciudadanos con la disposición crítica a objetar, refutar prácticas y ofrecer las posibilidades para que el hombre trascienda de la mera valoración de la realidad hacia niveles de reflexión que propicien la transformación de su propio entorno a través del uso de valores socialmente aceptados y reconocidos como lo son la convivencia democrática, la tolerancia y el reconocimiento del otro (Cassany, 2004; Jurado, 2008; Morales, 2017; Santiuste, 2001).

Estas propuestas educativas consiguen en la obra de Freire asidero teórico-práctico por el hecho de entrañar como pretensiones el despertar la conciencia del hombre y la facultad de reconocer los factores que han constituido socio-históricamente mecanismos de imposición. Frente a ello la pedagogía crítica ha propuesto la necesidad de tomar distancia como un acto cognitivo que le permita al sujeto identificar su objeto y, de manera objetiva y reflexiva su autoridad para generar cambios significativos en su ámbito de acción. Este compromiso implica trascender mediante el ejercicio permanente de habilidades del pensamiento crítico, instrumento que por su forma de operar le permite al sujeto emprender acciones intencionales que le lleven a juzgar todo lo que hace parte de su escenario de actuación inmediato, así como a revisarse en su proceder con el objeto de evitar la reproducción de acciones de dominación (Freire, 2002).

Esta actitud frente al mundo y sus semejantes, se interpreta como la asunción de una postura crítica capaz de elaborar juicios y razonamientos sobre la realidad, sobre su mundo, con la disposición intelectual de ofrecer alternativas y tomar de decisiones de elevado impacto social. De ello se infiere su compromiso con las necesidades propias de su entorno sobre el que es capaz de actuar combatiendo cualquier pretensión impositiva, mediante la construcción de escenarios para la comunicación y el democrático como condiciones que procuran la participación del otro, el intercambio de ideas y el diálogo como instrumentos necesarios para persuadir con razones fundadas en el respeto y la igualdad, así como con argumentos válidos y consensuados que favorezcan la coconstrucción de diversas maneras de ver la realidad (Arnaux, 2002).

Lo expuesto llevado al campo de la lectura, representa para Freire una herramienta con el potencial para llevar al pensamiento a operar en sus modos crítico y reflexivos, pretensión que persigue la preparación del sujeto para enfrentar el poder domesticador y el manejo impositivo de intereses y procesos ideológicos (Freire, 1991). En otras palabras, la lectura vista desde Freire puede entenderse como el medio idóneo para la construcción crítica del conocimiento, cometido del que se espera la asunción de una posición autónoma capaz de 
integrar diversos puntos de vista que evaluados con rigurosidad, permiten ofrecer aportes veraces, útiles y creíbles. Atendiendo a tal objetivo Freire (2010) propone el desarrollo de una sensibilidad especial que le coopere al sujeto con "la evaluación del contexto, lo que significa el reconocimiento de lo que viene sucediendo en él, cómo y por qué, premisas que se pueden lograr a través del uso de su pensar crítico" (p. 32).

En este sentido los postulados de Freire (2004) definen que la lectura exige "que el lector se comprometa con el texto, en una actitud capaz de desentrañar significados, verdades escondidas, intencionalidades y propósitos, para lo cual es necesario el pensamiento crítico que le permita llegar a lo más profundo" (p. 14). Todo esto implícitamente hace referencia a un lector revestido de una actitud rigurosa y una conciencia crítica como habilidades que favorecen hendir en la búsqueda de las razones que justifican y sustenten las afirmaciones de terceros, condición que desde la lectura crítica significa valorar profundamente el saber cómo punto de partida para la construcción crítica de su realidad (Jurado, 2008; Kincheloe, 2008; Kurland, 2003) partiendo de la profundización en las relaciones dadas en este espacio, de su dinámica y de sus problemas (Arias, 2015) como insumos necesarios para entender en profundidad así como ofrecer explicaciones innovadoras (Aisenberg, 2007; McLaren, 1994; Pérez, 2004).

Esta elaboración del mundo implica en palabras de Freire (2002) la existencia del "dialogo autentico, reconocimiento del otro y reconocimiento de si en el otro, es decisión y compromiso para colaborar en la construcción del mundo común" (p. 16). Desde el punto de vista social y educativo esta afirmación deja por sentado la invaluable función que la co-construcción de la realidad tiene dentro de los procesos de aprendizaje, debido a que en ella se encuentran comprometidas habilidades que favorecen la re-significación como operación mental que persigue elaboración de innovadoras y significativas apreciaciones (Zemelman, 2005). Por su parte Santelices (s/f) coincide con Freire al afirmar que la lectura crítica permite entre otras cosas "adquirir información adoptando una actitud crítica de tal manera que el lector sea capaz de descubrir problemas, contradicciones, limitaciones y argumentos que apoyen o refuten determinadas ideas o planteamientos y decidir racionalmente qué hacer o creer" (p. 1). Ello define que leer en modo crítico, entraña entre otras cosas la formación de un lector con una actitud irreverente capaz someter al juicio y al razonamiento y objetar al mundo y al conocimiento de manera recurrente como condiciones para asumir académica y científicamente posición.

Todo esto desde la educación liberadora, deja entrever el papel relevante que juega el introducir al sujeto en espacios abiertos a la divergencia de pensamiento, lo que en Freire es visto como un proceso que persigue la generación y transformación de la conciencia hacia niveles superiores, mediante la asunción de una postura que objetiva y objetadora del conocimiento, actitud que no debe ser valorada desde una connotación peyorativa, sino interpretada como condición elemental para consolidar el sentido crítico e irreverente que persigue revestir al lector de la autoridad para refutar acciones y afirmaciones científicas (Cassany, 2004; Freire, 2002) sin despreciar al que opina diferente, sino asumiéndolo con respeto y entendiendo su posición entraña otras visiones que sometidas al diálogo posibilitan el encuentro y la generación análisis reflexivos como actividades enriquecedoras del conocimiento científico (Sanmarti, 2011).

Para ello Freire entiende a la criticidad como una forma de promover el pensamiento divergente, objetivo y analítico desde el cual resignificar, construir nuevas perspectivas, elaborar juicios y maneras de ver el mundo, actividades mentales que por sus 
repercusiones llevan a romper con las posturas tradicionales que entrañan procesos opresivos que obligan a comprender las relaciones dadas en mundo de manera única. Frente a este lastre histórico, la pedagogía crítica ha planteado la transformación de la praxis educativa con la pretensión de formar para la libertad y la divergencia como herramientas que llevan a tomar una posición tolerante pero contestataria, que no deja de ser profunda, crítica y reflexiva, que persigue generar cambios y proceder con la convicción necesaria para enfrentar los mecanismos de dominación, así como la complejidad desde las más diversas visiones y perspectivas existentes sobre los fenómenos que emergen de la dinámica social (Daros, 2009).

De ello se infiere, el compromiso del hombre como sujeto dotado de un pensamiento activo que entraña entre otras cosas la capacidad para autorreflexionar y hacer uso de su conciencia para profundizar sobre las relaciones causales que históricamente han sumido a la sociedad en la más exacerbada dominación, inhabilitándola para pensar por sí misma y de manera divergente como requerimientos que para la educación del siglo XXI representan condiciones favorables de independencia, solidaridad y libertad, además de valores que deben mediar los procesos formativos que procuran motivar el compromiso y la integración del sujeto en la transformación social hasta lograr según Freire (1991) el "establecer la vinculación analítica entre lo que se lee y la realidad, lo que hace posible llevar a cabo un verdadero proceso de conocimiento transformador propio y del mundo" (p. 18).

Desde esta perspectiva, leer e investigar vienen a constituirse en medios idóneos cuyo potencial le permiten al hombre según Freire (2002) el "ejercer actos de compromiso social y el trascender los límites que le son impuestos por el mismo mundo hasta conseguir transformaciones significativas en un compromiso con el mundo, que debe ser humanizado para la humanización de los hombres" (p. 4). Ello indica que la lectura y la investigación además de fomentar procesos de exploración de la realidad, también propician la profundización en el conocimiento como una responsabilidad ciudadana que consiste en someter a revisión principios universales, de falsear ideas y de mover la conciencia del individuo hacia procesos reflexivos.

Todo esto desde el punto de vista educativo tiene repercusiones positivas, entre las que se destacan: la autonomía para expresar ideas propias, construir soluciones originales, da pie al pensamiento divergente, el cual a su vez tiene dentro de sus atribuciones el potencial de objetar, emitir juicios e inducir a la participación activa en procesos decisorios, que según López (2013) buscan el promover el desarrollo de capacidades tales como "opinar y manifestar el punto de vista personal con la fundamentación suficiente para revertir lógicamente ideas falaces en una actitud contestaría que intenta desvelar lo verdadero y cierto dentro del conocimiento científico" (p. 43).

En atención a lo expuesto, la lectura en Freire se entiende como una herramienta para problematizar situaciones emergentes de vínculos sociales, actividad que implica la continua y recurrente elaboración de interrogantes sobre los fenómenos suscitados en su realidad, en un intento por buscar respuestas y relaciones causales como insumos desde los cuales construir explicaciones profundas y rigurosas. Esta actitud desde el pensamiento crítico, amerita del desarrollo de un dialogo recursivo que derive en la resolución de contradicciones, la producción de aportes teóricos a partir de situaciones complejas que requieren de intervención, de cambio y transformación como actividades que persiguen mejorar la calidad de vida (Freire, 2002). 
Este posicionamiento implícitamente define, la repetida y constante disposición a indagar (Freire, s/f) habilidad que se vale de reflexión para mejorar la percepción y comprensión del mundo. De allí que, problematizar debe ser vista como la postura que asume el investigador y con tendencia consiste en percibir y captar la dinámica de su entorno, como punto de partida para transformarlo y, del mismo modo generar desde su compromiso social constituirse en un agente activo, autónomo y responsable capaz entender su posición en el mundo como punto de partida para el descubrimiento, la interacción y el dialogo con terceros que pudieran fungir como mediadores de su proceso de aprendizaje.

En atención a lo expuesto, problematizar como una actividad que permea la obra de Freire, integra dentro de sus bondades el estímulo de la indagación y la reflexión, herramientas que por su relevancia educativa son vistas como habilidades intelectuales que favorecen procesos creativos que permiten operar sobre el escenario social a través de acciones innovadoras. Para conseguir tales cometidos, las tendencias recientes sobre la lectura postulan que el lector debe involucrarse en actividades que promuevan el desarrollo de la criticidad, herramienta de transformación al servicio del pensamiento, que procura revestir al individuo de la sensibilidad y compromiso social (Freire, s/f) para proceder al servicio del colectivo como expresión de su empoderamiento, de la capacidad reflexiva y del pensamiento elaborado que le asiste para asumir responsablemente y con el compromiso como elemento mediador en la generación de mejores condiciones de vida presentes y futuras (Dewey, 1989).

\section{Lectura crítica desde la visión de Freire}

La lectura como proceso social e inherente a la producción de conocimiento científico, ha conseguido en Freire los fundamentos teóricos necesarios para entenderla como una herramienta que favorece el desarrollo de procesos de aprendizaje significativos, a partir de la construcción de nuevas maneras de ver el mundo y las relaciones que emergen del mismo. Ello significa entonces, que los aportes de la pedagogía crítica a la lectura giran en torno a la ampliación del sentido crítico como instrumento que permite diversificar las miradas sobre la realidad como requerimiento para trascender los reduccionismos teóricos que han históricamente han dominado el entendimiento de las relaciones sociales.

En atención a lo expuesto, es preciso comenzar afirmando que en Freire la concepción del proceso de alfabetización es compartida por tendencias recientes sobre la misma, que la conciben como un elemento mediador del aprendizaje en el siglo XXI. Ello significa que la alfabetización académica, se asume como la puerta de ingreso e integración del individuo a una nueva cultura, compuesta por un conjunto de prácticas y convenciones que giran en torno a la relación significativa del lector con el texto y su contex to (Freire, 1991; Fons, 2006) con el propósito de acercar al lector a los géneros académicos, a la producción de conocimiento y, a las maneras como las comunidades científicas presentan los hallazgos de sus indagaciones.

La apreciación de Freire sobre la lectura y en especial de la lectura desde la concepción critica, se evidencia en el hecho de posibilitarle al individuo la oportunidad de participar de manera democrática dentro del ámbito social. Ello implica, que la lectura es vista como una herramienta de empoderamiento y transformación que le posibilita al ciudadano ser capaz de comprometerse con la creación de condiciones en las que se interese no solo por entender el mundo y generar valoraciones, sino de crear juicios que trasciendan hacia la 
elaboración de esquemas racionales, principios y proposiciones que sirvan para explicar fenómenos y construir acciones de intervención con repercusiones sociales (Cassany, 2004; Daros, 2009; Freire, 2002; Pagès, 2009; Serrano, 2008) como evidencia de su sensibilidad social y académica que le asisten y que lo compromete con el contexto del que hace parte (Carlino, 2003; Freire, 2002).

Esta forma de entender a la lectura deja entrever su potencial para la construcción y la adhesión a valores democráticos, condición que demanda la necesidad de hacer a un lado el individualismo y el aislamiento por no representar condiciones favorables para la asunción de una posición crítica (Freire, 2002). Por tal motivo, se concibe a la interacción y la relación con el mundo como procesos mediadores y a su vez como requerimientos para ejercer influencia sobre la realidad de forma activa y con la responsabilidad de poner al servicio de la sociedad el uso del conocimiento como instrumento de transformación que favorezca la generación de cambios fundados en la reflexión, el análisis y la crítica como procesos necesarios para trascender hacia un accionar mediado por la autonomía y la originalidad (Ulloa, s/f). Lo expuesto indica que, la lectura además de permitirle al sujeto profundizar sobre su realidad para comprender su dinámica, también lo hace capaz de volverse participe de su re-significación como la manera de otorgarle fundamentos lógicos a los procesos de intervención colectiva y de indagación como habilidades sociales de significativo valor (Peña, 2007).

Para Freire (2004) la lectura crítica tiene dentro de sus cometidos

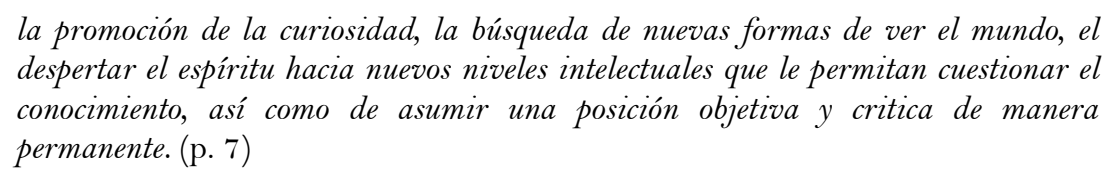

La lectura es entonces, la búsqueda inquieta de razones mediante el uso del pensamiento y la criticidad, habilidades desde las cuales ingresar rigurosamente al saber y a las diversas miradas existentes sobre su objeto de aprendizaje. En este sentido, la lectura además de mostrase como una herramienta que favorece la profundización en el conocimiento, también representa una de las maneras para promover la tolerancia y el reconocimiento del otro, aspectos que llevan al entendimiento de la dinámica social y de las relaciones históricas que han impedido su relación efectiva con otros y su liberación (Freire, 2002; Larrosa, 1998) así como la autonomía necesaria (Aisenberg, 2007) para construir y reconstruir nuevas visiones y maneras de actuar, dejando a un lado su actitud pasiva a la que se le atribuye su escasa capacidad para enfrentar la manipulación (Cassany, 2004; Prieto, 2008).

Todo ello puede ser asumido como una el resultado de la transformación experimentada por el individuo en su contacto con la realidad. Por lo tanto, es oportuno mencionar que Freire coincide con los aportes de la lectura crítica, al exponer que esta permite la formación ciudadana al favorecer que el lector comprenda la posición del otro, en una relación compartida que intenta la convivencia, el respeto y el hacer extensiva la transformación hacia terceros que hacen parte de su contexto (Freire, 2002). Esto es visto implícitamente como la evidencia de un nivel elevado de conciencia propiciados por el acercamiento crítico al conocimiento, proceso al que se le atribuye el ser propulsor del compromiso social y de generar actitudes democráticas centradas en el entendimiento del otro, así como en la creación de espacios que lo lleven a adherir a ese tercero tanto en la praxis como en el accionar de su propia libertad. 
Dicho de otra manera, la lectura crítica como promotora del entendimiento entre partes divergentes, persigue trascender del intercambio de ideas al compromiso de integrar al otro como parte integral del mundo, con el afán de propiciar el desarrollo de una conciencia capaz de percibir e ir más allá sorteando los obstáculos, superando los límites y las imposiciones que el conocimiento aportado por la realidad entraña, propósito que persigue el conseguir aportes informativos implícitos de los cuales depende la comprensión significativa del mundo (Alarcón y Fernández, 2006; Freire, 2002). En tal sentido, el lector crítico como actor social y dotado de una amplia visión de la realidad, debe buscar hallazgos en el conocimiento del pasado como punto de partida para analizar en prospectiva y hacerse un esquema mental que le permita anticiparse desde una postura crítica, reflexiva y autónoma a posibles situaciones futuras (Aisenberg, 2007; Morales, 2017; Serna y Díaz, 2015).

Para ello la lectura en su concepción crítica facilita la creación de un marco conceptual como aspecto favorable dentro del proceso valorativo de la realidad, debido a que permite la producción de apreciaciones sustentadas, de razonamientos sólidos y de aprendizajes significativos (Bazerman, 1988; Carrasco y Kent, 2011; Ulloa, s/f) como insumos desde los cuales aproximarse idónea y profundamente como operaciones al entendimiento de los pronunciamientos y posicionamientos científicos hechos por terceros (Freire, 2002). Por esta razón Freire asume el rol de la lectura como la propulsora del pensamiento crítico, instrumento del cual depende la elaboración de aportes reflexivos que le posibilitan al lector operar con seguridad y libertad teniendo como pretensión de romper con la dependencia inculcada históricamente y a la cual se le han hecho atribuciones negativas como las de imposibilitar el cambio y transformación.

Esta premisa el lector es visto como agente activo capaz de usar la lectura como medio para asumir una actitud crítica y reflexiva, con la madurez suficiente para ver en el conocimiento y el mundo lo que es útil, comprobable y necesario de aquello que es superfluo. Interpretando a Freire, la lectura en modo crítico permite que el individuo consiga realizar disertaciones, diálogos y pronunciamientos coherentes que den cuenta de la transformación vivenciada como resultado del interactuar con su propio contex to y con la información (Fons, 2006; Freire, 2002; Peppino, 2006) operaciones que favorecen el desarrollo de la conciencia social necesaria para hacer uso de su pensamiento en modo divergente y autónomo. Esta posición es compartida por Alarcón y Fernández (2006) quienes asumen que el lector critico debe ver en la lectura "una herramienta para profundizar y ampliar los conocimientos y la capacidad aportar significativamente otras áreas del mundo social y académico" (p. 4).

Esta actitud implica, que el lector además de ir más allá en su proceder frente al conocimiento, debe sentirse identificado con su contexto al intentar con su actuar la búsqueda del bien común en una actitud empática que persigue llevar a la sociedad a ser más humana, participativa y equitativa, como resultado de la sensibilidad aportada por su interacción con el conocimiento y con el medio social (Freire, s/f; Martínez, 2004). Ello indica que los recurrentes cambios sociales exigen del lector el ejercitar sus habilidades sociales y sus competencias críticas para actuar en forma coherente y oportuna, adentrándose en el manejo de la crisis y ofreciendo alternativas que permitan sortear los obstáculos y vicisitudes que se le presentan, para lo cual va a requerir de una disposición reflexiva que lo lleve a actuar de manera innovadora y creativa (Freire, 2004). 
Lo expuesto lleva deducir que leer en modo crítico representa un proceso intelectual caracterizado por la asunción de un compromiso formativo, capaz de profundizar sobre el conocimiento existente o acumulado (Zemelman, 2005) sobre el cual opera con el propósito de detectar ideas subyacentes y posturas ideológicas que determinan la asunción de una u otra posición frente al saber y, de las cuales va a depender la construcción de nuevas apreciaciones con profundas repercusiones en el accionar transformador del mundo. En palabras de Freire (2002) el resultado de esta actitud crítica no es otro que "llevar al sujeto a asumir una posición capaz de objetar activamente el conocimiento, deslastrándose de su conciencia ingenua como requisito para interactuar en un compromiso auténtico que le permita transformar e incidir de manera significativa sobre su contexto" (p. 5).

En este mismo orden de ideas, otras perspectivas de la pedagogía crítica coinciden con Freire al asegurar que la lectura debe ser vista como un proceso que favorece el aprendizaje de múltiples e innovadoras de entender la dinámica social, pero además como una herramienta que hace posible ingresar al conocimiento y a la información provechosamente a través de un dialogo interactivo entre el lector, el autor y el contexto (Prieto, 2008; Ulloa, s/f) en el que el primero busca desentrañar la tesis, las ideas y los argumentos usados por el segundo para sostener su posición frente al tema que aborda. Para Freire (2004) este modo de hacer lectura, constituye la posibilidad de crear condiciones para que el sujeto haga uso del conocimiento desde un enfoque social que le permita "lograr la igualdad, transformar e incluirse dentro de procesos de mejoramiento de la humanidad, con el objeto de sentar los fundamentos para liberar a otros y articular esfuerzos de manera colectiva” (p. 2).

Aunado a ello, apreciaciones sobre el pensamiento reflexivo coinciden al afirmar esta manera de hacer lectura consiste en un proceso constructivo en el que el lector establece una relación profunda y recursiva con el conocimiento, a lo que Daros (2009) define como "un ir y venir sobre la información, en una actitud seria que le permite buscar la verdad, verificar las premisas sobre las que se sustenta el conocimiento y, a partir de allí generar soluciones que trasciendan a otros escenarios sociales" (p. 35). Este énfasis en la comprensión crítica hace referencia a las competencias críticas que lector debe manejar para realizar formulaciones que trasciendan lo teórico para convertirse en acciones prácticas cuyo alcance social originen transformaciones reales y significativas (Freire, 2004; Morales, 2017).

Frente a estas demandas sociales Hawes (2003) del lector crítico se espera el despliegue de estrategias para "manejar y buscar información con el propósito de localizar e ingresar al conocimiento como medios para construir alternativas que favorezcan la resolución de problemas" (p. 14). Ello indica que la lectura crítica dentro de sus atribuciones cuenta con el posibilitar la valoración profunda y acuciosa del conocimiento, procesos que desde el aprendizaje significativo son necesarios para producir ideas rigurosas y relevantes (Díaz, 2015). Por su parte Santelices (s/f) coincide con Freire al esgrimir que la lectura le aporta al desarrollo del pensamiento crítico aspectos importantes tales como: la posibilidad de problematizar la realidad en búsqueda de razones que justifiquen la existencia de determinadas situaciones; la capacidad para elaborar construcciones teóricas a partir de la evaluación y el análisis de ideas; el potencial para producir ideas innovadoras como aportes teóricos a través de los cuales conseguir la resolución lógica y coherente de problemas sociales, y finamente, buscar relaciones causales dentro del conocimiento con el propósito 
de dar con la verdad a través del cuestionamiento del saber acumulado históricamente (Zemelman, 2005).

Desde la posición de Freire (2004) se trata de preparar al sujeto para que "se interese en adentrarse profundamente en el conocimiento, en la comprensión de los hechos, en el análisis de posiciones antagónicas, en la búsqueda de soluciones a problemas desde diversas perspectivas mediante el uso de la criticidad” (p. 9). Ello requiere del lector un elevado nivel de compromiso académico e intelectual, capaz de hacer uso de sus competencias para construir ideas y, a su vez transferirlas con autenticidad al campo de la praxis social como escenario al que debe abocarse para realizar mejoras significativas que trasciendan en el tiempo y garanticen el anhelado bienestar colectivo.

En consecuencia, los postulados de Freire posicionan a la lectura como parte medular de la educación de todos los tiempos y como un proceso cognoscitivo encausado hacia la liberación, cometidos que demandan el acompañamiento y la guía constante que prepare al lector para la vida (Fons, 2006; Márquez y Prat, 2005; Torres, 2006). Ello implica que la lectura guiada debe ser vista como una herramienta al servicio del acercamiento al conocimiento, pues la misma favorece que el lector se inicie significativamente en la construcción de nuevos aprendizajes que le lleven a apropiarse de las prácticas y convenciones propias de cada disciplina, así como de la forma como se organiza y estructura el conocimiento y la información (Carlino, 2002). Esta intervención del docente implica que el acompañamiento puede ser visto como una estrategia que el docente puede manejar para ir estrechando la elaboración de interpretaciones sólidas lo más ajustadas posible a los textos (Aisenberg, 2007).

\section{Esquema de lectura crítica propuesto por Freire}

El esquema que se presenta a continuación, intenta aproximarse a la propuesta de lectura crítica que permea gran parte de las obras de Freire. El mismo sigue la siguiente estructura:

- La lectura de la realidad, para Freire el sujeto antes de comprender el código escrito pasa por la lectura del mundo, del cual toma los primeros referentes que constituirán sus representaciones mentales de la dinámica, funcionamiento e interacción entre los objetos y sujetos que hacen parte de su contexto.

- La búsqueda inquieta de relaciones, vínculos y relaciones causales constituyen el siguiente proceso que experimenta el lector y, que según Freire responde a una expresión de su pensamiento crítico que le insta a establecer un diálogo profundo y acucioso con su espacio de convivencia y con los que hacen parte del mismo.

- La identificación de contradicciones, hace referencia a un nivel más elevado de criticidad que persigue dilucidar las inconsistencias lógicas contenidas en la información con la que tiene contacto y que representan formas reduccionistas de ver el mundo. En este aspecto Freire coincide con los postulados de la lectura crítica como aquella actividad que involucra habilidades cognitivas enfocadas en valorar la veracidad, consistencia lógica y credibilidad de la información.

- La construcción de marcos de referencia en los que el lector integra los elementos teóricos y conceptuales como fundamentos que le sirven para apoyar 
la elaboración pronunciamientos consistentes y propias opiniones lógicas como elaboraciones su pensamiento autónomo y divergente, cualidades que aluden a un sujeto inmerso en procesos de transformación.

- La generación de nuevos aprendizajes como resultado de la interacción profunda con la realidad, condición de la que se derivan acciones de intervención que procuran la participación del sujeto en la resolución de problemas colectivos, actitud que refiere a su sensibilidad social y a la generación de cambios en su contexto.

- Como resultado del desarrollo de sus modos de pensamiento crítico y divergente, el lector viene a constituirse en constructor de ideas innovadoras a partir de las cuales accionar de manera independiente y responsable como operaciones recurrentes que le asisten en la búsqueda de mayores niveles de autonomía frente a los intentos de manipulación e imposición ideológica.

\section{La investigación en Freire}

La propuesta pedagógica de Freire y de la pedagogía crítica en general, tiene como enfoque ofrecer los medios y condiciones necesarias para contribuir con la formación ciudadana con sentido de pertenencia, reflexiva, libre y capaz de llevar a cabo procesos de indagación que le permitan descubrir nuevas maneras de apreciar el mundo. Esta premisa indica que, la investigación como actividad que transversaliza a la educación como proceso de liberación, implica el desarrollo de competencias y habilidades científicas que involucran al pensamiento como instrumento desde el cual construir un discurso válido, riguroso y coherente con las necesidades sociales.

Este cúmulo de exigencias hace referencia a un sujeto revestido de la sensibilidad para problematizar a partir de los fenómenos suscitados en su propia realidad y en cual identifica sus componentes, relaciones y repercusiones, en un intento por ofrecer apreciaciones propias rompan con posiciones científicas monádicas y tradicionales. Tal manera de abordar el mundo según Freire (2004) es vista como actitud que persigue entre otras cosas "la observación imparcial, objetiva y segura de los hechos y acontecimientos como parte de su proceder ético y científico” (p. 16).

En este sentido la investigación en Freire es vista como un proceso que estimula y favorece el descubrimiento y la curiosidad como actividades intelectuales cuya importancia se centrada en posibilitarle al sujeto acercamientos críticos a la realidad con el propósito de percibir intelectualmente su dinámica, operaciones que entrañan la identificación de relaciones causales, actores involucrados e implicaciones, lo que significa someter su fenómeno de estudio a procesos de revisión profunda cuyo resultados lleven a la producción de conocimiento nuevo (Alarcón y Fernández, 2006; Freire, s/f).

De esta manera, la investigación como actividad inherente a la formación de un pensamiento elaborado según Freire (2002) tiene como propósito el "estimulo del pensamiento autentico, pensamiento que no se deja confundir por las visiones parciales de la realidad, buscando, por el contrario, los nexos que conectan uno y otro punto, uno y otro problema" (p. 54). Esta actitud implícitamente hace referencia a un proceso minucioso que se enmarca en el estudiar la realidad desde una posición sistémica que persigue integrar la relación de cada situación con otras, entendiéndola como un todo y a su vez fragmentada en componentes sobre las que existen indefectiblemente nexos. 
En este sentido, del investigador como agente crítico se espera la construcción innovadora de aportes científicos derivados de su experiencia con la realidad y del ejercicio de acciones reflexivas recurrentes como operaciones mentales que procuran trascender los actos reproductivos y la repetición los resultados de discusiones, hasta construir por sí mismo su propia manera de ver la realidad y, como consecuencia favorecer la búsqueda de la verdad, evitando con ello la imposición de concepciones, razonamientos y apreciaciones carentes de veracidad (Ulloa, s/f). Tal compromiso hace referencia al investigador como lector de la realidad, cuya disposición valorativa le posibilita para determinar los fundamentos sobre los que se sustentan razonamientos o juicios expuestos en la información aportada históricamente para determinar su credibilidad (Arnaux, 2002).

De ello se infiere según Freire (2002) el verdadero rol del investigador con respecto a la realidad, el cual se centra en "buscar aprehenderla en su riqueza, en su significado, en su pluralidad, en su devenir, en su constitución histórica" (p. 80). Este accionar sobre el mundo hace referencia a un proceso activo que se vale de la criticidad y de la reflexión como habilidades de uso recurrente (Martínez, 2012) que impulsan al investigador a ir más allá de lo que se pueda preciar superficialmente, hasta profundizar, aprehender e identificar aspectos sobre los cuales elaborar explicaciones y ofrecer soluciones coherentes y con la relevancia oportuna (Alarcón y Fernández, 2006; Blaxter, Hughes y Tight, 2002; Freire, 2002; Prieto, 2008).

Este conjunto de competencias científicas es vistas en Freire como medios encargados de orientar el quehacer del investigador, quien es capaz de comprender la totalidad de los elementos incursos, relacionándolos directa e indirectamente y dejando ver sus vínculo y la interdependencia como resultado de su análisis profundos y de la formación crítica que le aportan la experticia para dar cuenta de su entorno. Lograr tales cometidos demanda del investigador según Freire (2002) el lograr la "escisión de la situación figurada que posibilita el descubrir la interacción entre las partes y el todo escindido" (p. 89).

Lo expuesto indica que el investigador se constituye en un observador crítico que aprecia no solo los cambios que sufren los objetos de estudio, sino además de las transformaciones que sus propias precepciones van sufridas a lo largo de su acercamiento a estos. Esta disposición implica el uso del análisis y de la interpretación como actividades mentales que hacen posible entender los diversos estadios por los que ha pasado su fenómeno. Este proceder implícitamente hace referencia a la disposición académica de la que depende la revisión de su propio proceso reflexivo y sistemático, que le lleva a verificar la evolución que sufre su formación como investigador, condición que demanda el hacer revisión introspectiva para determinar los cambios experimentados y, como consecuencia de ello agudizar sus habilidades para valorar significativamente los fenómenos de los que se ocupa.

Este acercamiento objetivo a la comprensión del mundo, demanda la identificación de las relaciones emergentes como punto de partida para la producción de nuevos planteamientos que vienen a constituirse en reconstrucciones significativas del conocimiento que se deben a un acto cooperativo (Freire, 2004) que intenta a su vez transformar a los actores que hacen parte de su proceso investigativo. En Freire lograr tales pretensiones exige del investigador la elaboración de criterios que dirijan el acercamiento y la valoración de su objeto, operaciones mentales que integran aspectos como la rigurosidad, sistematicidad y el uso de la criticidad para captar conclusiones 
coherentes y lógicas (Campanario y Moya, 1999; Corrado y Eizaguirre, 2003; Dewey, 1989; Freire, 2002; Konrr, 2010; Martínez, 2004; Pagès, 2009).

En consecuencia, la propuesta de Freire sobre la investigación científica debe ser considerada con detenimiento, debido a que se asume desde dos perspectivas relacionadas, por un lado como actividad académica y por el otro como una herramienta social, que pretende llevar al investigador a la construcción de ideas cercanas a la verdad, una verdad co-construida o elaborada socialmente, que intenta dejar en evidencia las contradicciones e inconsistencias existentes en el conocimiento dado y en las posiciones que intentan erigirse como verdades absolutas. Ello se traduce como el accionar sobre la realidad haciendo uso de la problematización como habilidad cognitiva de la que depende el entendimiento de las relaciones entretejidas en los conflictos sociales (Freire, 2002) ante lo que el investigador según expone Daros (2009) debe asumir una postura dada a "la construcción reflexiva y abstractiva de la experiencia” (p. 6).

\section{Esquema de investigación propuesto por Freire}

Una de las herramientas al servicio de la formación crítica y del pensamiento divergente lo constituye la investigación, idea que en Freire representa la manera en que el sujeto establece contacto con el mundo a través de la problematización y valoración de sus experiencias como la manera de comprender aquello que aprecia conflictivo, complejo e interesante. Desde esta perspectiva, investigar tomando como referente la pedagogía crítica, consiste en formular de manera responsable una serie de planteamientos a partir de la comprensión reflexiva de los hechos, postura que implica dejas a un lado la reproducción ingenua para trascender a producción de conocimiento.

En atención a lo expuesto Freire (2004) propone que la investigación como proceso científico, requiere de un elevado nivel de compromiso intelectual que le permita "comparar situaciones, constatar la veracidad de sus hallazgos y evaluar las premisas sobre las que se sustentan las afirmaciones propias y las de terceros, operaciones que facilitan la elaboración de conclusiones veraces" (p. 20). Atendiendo a tales cometidos, se describen algunas ideas que se acercan al esquema de investigación propuesto por Freire:

- Observación de la realidad, para Freire esta fase le permite al investigador apreciar a través de los sentidos todo lo que sucede en su entorno, asumiendo para ello una actitud crítica de la que depende la detección de situaciones que satisfagan su curiosidad.

- Delimitar su contexto de estudio constituye para Freire definir la situación de estudio con precisión y atendiendo a aspectos como la relevancia y la pertinencia social.

- La escogencia de un tema generador consiste en la valoración crítica de una situación preferiblemente de su propio contexto, condición que permitirá aproximarse, estudiar y entender con mayor facilidad su fenómeno de estudio.

- El proceso de problematizar en Freire no es más que revisión profunda de una situación con la que se interactúa y establece un diálogo que persigue apropiarse de su funcionamiento, dependencia histórica, relaciones causales y posibles efectos que se generarán a partir de su permanencia en el tiempo. 
- La construcción dialógica de su experiencia, este proceso de apoya en la sistematización y organización del conocimiento derivado de su convivencia con la situación objeto de estudio, así como de los aportes de terceros que cooperaron en el proceso de investigación.

- Como resultado de lo anterior, Freire propone que el potencial de la investigación se desprende de la dinámica que el sujeto establezca entre la acción y la reflexión, operaciones que a su vez son contentivas del poder transformador necesario para cambiar la realidad.

\section{Conclusiones}

Entender la formación integral del hombre desde la perspectiva de Freire, exige la revisión de sus propuestas teóricas, cuya vigencia permanece intacta al ser aceptada elevado compromiso con el desarrollo y la transformación del ser humano. Esta condición se aprecia en planteamientos que dejan ver la necesidad de crear condiciones educativas en las que el ciudadano logre maximizar su potencial cognitivo e intelectual, requerimientos coyunturales sobre los que cimienta su función social al fomentar el sentido de pertenencia y la inserción en actividades transformadoras que lo lleven a participar de manera autónoma, activa y desde un compromiso reflexivo que derive en la construcción de condiciones de vida equitativas y apegadas el bien común (Freire, 2002).

En este sentido, la propuesta educativa de Freire deja ver a la formación ciudadana como ideal que persigue humanizar al hombre, proceso en el que la lectura y a la investigación constituyen medios que hacen posible el operar sobre la realidad desde una actitud exploratoria y curiosa en la que participan otras habilidades propias del pensamiento crítico como la valoración, la comprensión y la reflexión que le permiten al sujeto establecer conexiones lógicas y explicativas de las relaciones que se dan en su espacio de convivencia.

Por ende, la lectura crítica y la investigación en Freire responden a actividades científicas al servicio de la liberación, debido que estas entrañan entre otras cosas el desarrollo de una actitud crítica y responsable como operaciones mentales que coadyuvan con la construcción de apreciaciones e interpretaciones y sólidas sobre la realidad y la información. Todo esto hace referencia lo propone la teoría crítica, a un sujeto con las competencias para explorar, valorar, elaborar juicios y producir razonamientos que den cuenta de un pensamiento autónomo capaz de objetar el mundo en búsqueda de explicaciones y respuestas a partir de las cuales diseñar acciones de intervención social generadoras de cambio y transformación.

Por consiguiente, leer e investigar en Freire constituyen medios de reflexión que le permiten al sujeto el comprender en profundidad el mundo y las relaciones dadas en el mismo. Esto en palabras de López (2013) consiste en entender que "la investigación y la lectura favorecen en el individuo el desarrollo de habilidades cognitivas como el problematizar, el juzgar la credibilidad y fiabilidad de las afirmaciones históricamente tomadas como ciertas, así como formular hipótesis, comprobarlas y generar conclusiones" (p. 44). Parafraseando a Freire este proceso es el resultado de la interacción profunda con el conocimiento en la que el lector es capaz de "realizar construcciones significativas que le permitan trascender hacia mayores niveles de comprensión que abran la brecha para generar verdaderos cambios" (p. 4). 
En síntesis, la lectura y la investigación en Paulo Freire se asumen como herramientas de transformación del pensamiento, cuyas repercusiones determinan el accionar crítico del hombre sobre el mundo, posibilitando entre otras cosas: tomar conciencia a través del uso de la reflexión como habilidad que le reviste de autoridad para combatir arbitrariedades; desarrollar competencias críticas que le comprometan con el rompimiento de los esquemas impositivos de dominación histórica, proceso a partir del cual va a depender la construcción una identidad propia que le inste a conseguir la transformación propia y de terceros que hacen parte de su realidad.

\section{Referencias}

Aisenberg, B (2007). Ayudar a leer en sociales. Revista Quehacer educativo, 83, $42-45$.

Argudin, L. M. (1995) Aprendiendo a pensar leyendo bien. Ciudad de México: Plaza y Valdés Editores.

Arias, D. (2015). La enseñanza de las ciencias sociales en Colombia: Lugar de las disciplinas y disputa por la hegemonía de un saber. Revista de Estudios Sociales, 52, 134-146. https://doi.org/10.7440/res52.2015.09

Alarcón P. y Fernández P. (2006). La lectura crítica como herramienta básica en la educación superior. Revista Graffylia, 6, 46-55.

Arnaux, E. (2002). La lectura y la escritura en la universidad. Buenos Aires: Universidad de Buenos Aires.

Bazerman, C. (1988). The problem of writing knowledge. En W. Connolly (Ed.), Shaping writte knowledge. The genre and activity of the experimental article in science (pp. 4-17). Madison, WI: The University of Wisconsin Press.

Blaxter, L., Hughes, C. y Tight, M. (2002). Cómo se hace una investigación. Ciudad de México: Editorial Gedisa.

Campanario, J. M. y Moya, A. (1999). ¿Cómo enseñar ciencias? principales tendencias y propuestas. Investigación Didáctica, 17(2), 179-192.

Carlino, P. (2002). Leer, escribir y aprender en la universidad: cómo lo hacen en Australia y por qué. Revista del Instituto de Investigaciones de la Facultad de Psicología, 7(2), 57-78.

Carlino, P. (2003). Alfabetización académica: Un cambio necesario, algunas alternativas posibles. Educere, 6(20), 409-420.

Carrasco, A. y Kent, R. (2011). Leer y escribir en el doctorado o el reto de formarse como autor de ciencias. Revista Mexicana de Investigación Educativa, 16(51), 1227-1251.

Cassany, D. (2004). Tras las líneas. Sobre la lectura contemporánea. Barcelona: Ediciones Anagrama.

Corrado, R. y Eizaguirre, M. (2003). El profesor y las prácticas de lectura en el ámbito universitario. Revista Iberoamericana de Educación, 30, 1-8.

Daros, W. (2009). Teoría del aprendizaje reflexivo. Argentina: Editorial RICE.

Delors, J. (1994). Los pilares de la educación del futuro. Revista Colombiana de Sociología, 25, 11-23.

Dewey, J. (1989). ¿Cómo pensamos? Nueva exposición de la relación entre el pensamiento reflexivo y proceso educativo. Ciudad de México: Paidós.

Díaz, P. (2015). La lectura crítica y su relación con la formación disciplinar de estudiantes universitarios. Revista de la Educación Superior, 44(4), 139-158. https://doi.org/10.1016/j.resu.2015.12.006 
Fons, M. (2006). Enseñar a leer para vivir. Barcelona: Universidad de Barcelona

Freire, P. (s/f). Educación y cambio. Recuperado de http://derechoepja.org/wpcontent/uploads/2015/05/freire-paulo_educacicion-y-cambio.pdf

Freire, P. (1967). Educación como práctica de la libertad. Ciudad de México: Siglo XXI.

Freire, P. (1991). La importancia de leer y el proceso de liberación. Ciudad de México: Siglo XXI.

Freire, P. (2002). Pedagogía del oprimido. Buenos Aires: Siglo XXI.

Freire, P. (2004). Pedagogía de la autonomía. Sao Paulo: Paz e Terra SA.

Freire, P. (2005). La importancia de leer y el proceso de liberación. Ciudad de México: Siglo XXI.

Freire, P. (2010). Cartas a quien pretende enseñar. Buenos Aires: Siglo XXI.

Goodman, K. (1996). La lectura, la escritura y los textos escritos: Una perspectiva transaccional sociopsicolingüística. Textos en Contexto, 2, 78-89.

Hawes, J. (2003). Pensamiento crítico en la formación universitaria. Santiago de Chile: Universidad de Talca.

Kincheloe, J. (2008). La pedagogía critica en el siglo XXI: Evolucionar para sobrevivir. Barcelona: Graó.

Konrr, P. (2010). Estrategias para el abordaje de textos. Material didáctico de apoyo a la enseñanza de las ciencias sociales. Buenos Aires: Universidad Nacional del General Sarmiento.

Kurland, D. (2003). Lectura crítica versus pensamiento crítico. Cali: Eduteka.

Jurado, F. (2008). Formación de lectores críticos desde el aula. Revista Iberoamericana de Educación, 46, 89-105.

Larrosa, J. (1998). La experiencia de la lectura. Estudios sobre literatura y formación. Barcelona: Editorial Laertes.

López, G. (2013). Pensamiento crítico en el aula. Docencia e Investigación, 37(22), 41-60.

Márquez, C. y Prat, A. (2005). Enseñanza en clase de ciencias. Innovaciones Didácticas, 23(3), 431440.

Martínez, M. (2004). Ciencia y arte en la metodología cualitativa. Ciudad de México: Editorial Trillas.

Martínez, A. (2012). Verdades y mentiras sobre la escuela. Bogotá: Idep.

McLaren, P. (1994). Pedagogía crítica y cultura depredadora. Cali: Eduteka

Morales, J. (2017). Pensamiento crítico y lectura en ciencias sociales. Revista Electrónica Calidad en la Educación Superior, 8(2), 265- 282.

Pagès, J. (2009). Enseñar y aprender ciencias sociales en el siglo XXI: Reflexiones casi al final de una década. Medellín: Universidad de Antioquia.

Peña, L. (2007). El proyecto: Leer y escribir en la universidad. Bogotá: Pontificia Universidad Javeriana.

Peppino, M. (2006). Lectura y pensamiento críticos: Estrategias para desenvolverse en el ciberespacio. Ciudad de México: Universidad Autónoma de México.

Pérez, M. (2004). Leer, escribir, participar: Un reto para la escuela, una condición de la política. Lenguaje, 32, 71-88.

Prieto, J. (2008). Pensamiento crítico y universidad: Estrategias para la consolidación de una sociedad democrática en México. Revista Investigación y Ciencia. Universidad de Aguascalientes. 42(4), 36-42. 
Sanmarti, N. (2011). Leer para aprender ciencias. Recuperado de http://leer.es/documents/235507/242734/art_prof_eso_leerciencias_neussanmarti.pdf/b 3507413-ca58-4a00-bf37-c30c619b627f

Santelices, L. (s/f). Desarrollo del pensamiento crítico: su relación con la comprensión de la lectura y otras áreas del currículo de educación básica. Santiago de Chile: Pontificia Universidad Católica de Chile.

Santiuste, V. (2001). Quelques réflexions sur la valur éducative de la Philosophie. Bruselas: Seminario de Filosofía.

Serna, J. y Díaz, J. (2014). Propuesta didáctica para la comprensión crítica en la Universidad La Gran Colombia. Cuadernos de Lingüística Hispánica, 25, 165-180.

Serrano, S. (2008). El desarrollo de la comprensión crítica en los estudiantes universitarios: hacia una propuesta didáctica. Educere, 12(42), 505-514.

Smith, F. (1983). Comprensión de la lectura. Ciudad de México: Editorial Trillas.

Solé, I. (1997). Estrategias de lectura. Barcelona: Editorial GRAÓ.

Torres, R. (2006). Alfabetización y aprendizaje a lo largo de toda la vida. Revista Interamericana de Educación de Adultos, 1, 1-13.

Ulloa, J. (s/f). La lectura y la escritura ¿Se deben aprender en la universidad? Programa de Formación de Académicos, 10, 1-7.

Valera, H y Escobar, M. (s/f). Introducción de la importancia de leer y el proceso de liberación. Recuperado de http://ceiphistorica.com/wp-content/uploads/2015/12/Paulo-Freire-Laimportancia-de-leer-y-el-proceso-de-liberaci\%C3\%B3n.pdf

Zemelman, H. (2005). Voluntad de conocer. Barcelona: Anthropos.

\section{Breve CV del autor}

\section{Jesús Morales}

Politólogo y Abogado de la Universidad de Los Andes (ULA). Magister en Educación Mención Orientación Educativa por la Universidad Pedagógica Experimental Libertador (UPEL). Magister en Educación Mención Lectura y Escritura por la Universidad de Los Andes (ULA). Candidato a Doctor en Antropología. Docente de pregrado de las Escuelas de Criminología y Derecho de la Universidad de Los Andes (ULA). Docente del Programa de Actualización Docente de le Universidad de Los Andes (ULA). Líneas de Investigación: orientación educativa, lectura y escritura académicas y violencia en escenarios escolares. Investigador reconocido por el Programa de Estímulo a la Investigación de la Universidad de Los Andes (ULA). ORCID ID: https://orcid.org/0000-0002-8756-5420. Email: lectoescrituraula@gmail.com 